\title{
A High-Throughput Disease-Specific Phenotype Detection System of Parkinson's Disease
}

\author{
AKIHIRO YAMAGUCHI*1), KEI-ICHI ISHIKAWA*1) 2), SHINJI SAIKI*2), \\ NOBUTAKA HATTORI*2), WADO AKAMATSU*1) \\ *1) Center for Genomic and Regenerative Medicine, Juntendo University Faculty of Medicine, Tokyo, Japan, \\ *2) Department of Neurology, Juntendo University Faculty of Medicine, Tokyo, Japan
}

\begin{abstract}
Disease-specific induced pluripotent stem cells (iPSCs) are widely used for disease modeling and drug discovery. We have established iPSCs from 7 types of familial Parkinson's disease (PD). Notably, each form of familial PD has specific cellular functional abnormalities that induce extensive loss of dopaminergic neurons. To accelerate PD-iPSC research, we sought to establish a 96-well based high-throughput neural differentiation and phenotype detection system. iPSCs were differentiated into dopaminergic neurons on the 96-well plates by a neurosphere-based differentiation protocol (Stem Cell Reports, 2016). Subsequently, several PD-related phenotypes, including impaired mitophagy, which is considered a major phenotype in PARK2 (autosomal recessive juvenile PD, caused by mutations in PRKN gene) and PARK6 (autosomal recessive juvenile PD, caused by mutations in PINK1 gene), cell vulnerability, and $\alpha$-synuclein accumulation commonly seen in PD, were quantified automatically by using In Cell Analyzer 2200 and In Cell Developer Toolbox (GE Healthcare). We successfully detected the phenotypes characterized in each form of familial PD as compared with healthy controls both quantitatively, and reproducibly. By using this high-throughput assay system, we screened a library of 320 compounds to evaluate phenotypic recovery. We identified several compounds that modify impaired mitophagy observed in PARK2 and PARK6 dopaminergic neurons. Furthermore, we established a small scale iPSC induction method, and completed iPSC induction from over 100 sporadic PD patients. By applying both the high-throughput neural differentiation method and phenotype detection system, we are trying to capture abnormal phenotypes in sporadic PD patients. Thus, this high-throughput phenotype detection system could comprehensively compare phenotypes of each form of familial PD, as well as perform drug screening. This approach is applicable for sporadic cases and other neurodegenerative diseases.
\end{abstract}

Key words: Parkinson's disease, iPSCs, high-throughput, disease modeling, drug discovery

Corresponding author: Wado Akamatsu

Center for Genomic and Regenerative Medicine, Juntendo University Faculty of Medicine

2-1-1 Hongo, Bunkyo-ku, Tokyo 113-8421, Japan

TEL: +81-3-5802-1728 (ext. 3821) E-mail: awado@juntendo.ac.jp

345th Triannual Meeting of the Juntendo Medical Society: Medical Research Update〔Held on May 19, 2018〕

〔Received July 27, 2018〕〔Accepted Nov. 15, 2018〕

Copyright (C) 2019 The Juntendo Medical Society. This is an open access article distributed under the terms of Creative Commons Attribution License (CC BY), which permits unrestricted use, distribution, and reproduction in any medium, provided the original source is properly credited. doi: 10.14789/jmj.2019.65.JMJ18-R11 